\title{
The fresnel interferometric imager
}

\author{
Laurent Koechlin • Denis Serre • Paul Deba · Roser Pelló • \\ Christelle Peillon • Paul Duchon • Ana Ines Gomez de Castro • \\ Margarita Karovska • Jean-Michel Désert - David Ehrenreich • \\ Guillaume Hebrard • Alain Lecavelier des Etangs • Roger Ferlet • \\ David Sing • Alfred Vidal-Madjar
}

Received: 31 January 2008 / Accepted: 1 July 2008

(C) Springer Science + Business Media B.V. 2008

\begin{abstract}
The Fresnel Interferometric Imager has been proposed to the European Space Agency (ESA) Cosmic Vision plan as a class L mission. This mission addresses several themes of the CV Plan: Exoplanet study, Matter in extreme conditions, and The Universe taking shape. This paper is an abridged version of the original ESA proposal. We have removed most of the technical and financial issues, to concentrate on the instrumental design and astrophysical missions. The instrument proposed is an ultra-lightweight telescope, featuring a novel optical concept based on diffraction focussing. It yields high dynamic range images, while releasing constraints on positioning and manufacturing of the main optical elements. This concept should open the way to very large apertures in space. In this two spacecraft formation-flying instrument, one spacecraft holds the focussing element: the Fresnel interferometric array; the
\end{abstract}

L. Koechlin $(\varangle) \cdot$ D. Serre · P. Deba · R. Pelló · C. Peillon

Laboratoire d'Astrophysique de Toulouse-Tarbes,

Université de Toulouse, CNRS, 31400 Toulouse, France

e-mail: laurent.koechlin@ast.obs-mip.fr

P. Duchon

Centre National dEtudes Spatiales (CNES), 31400 Toulouse, France

\section{A. I. Gomez de Castro}

Fac. de CC Matematicas, Universidad Complutense de Madrid, 28040 Madrid, Spain

M. Karovska

Harvard Smithsonian Center for Astrophysics (CfA), Cambridge, MA 02138, USA

J.-M. Désert · D. Ehrenreich · G. Hebrard · A. Lecavelier des Etangs ·

R. Ferlet · D. Sing · A. Vidal-Madjar

Universite Pierre \& Marie Curie, CNRS, 75014 Paris, France 
other spacecraft holds the field optics, focal instrumentation, and detectors. The Fresnel array proposed here is a $3.6 \times 3.6 \mathrm{~m}$ square opaque foil punched with $10^{5}$ to $10^{6}$ void "subapertures". Focusing is achieved with no other optical element: the shape and positioning of the subapertures (holes in the foil) is responsible for beam combining by diffraction, and $5 \%$ to $10 \%$ of the total incident light ends up into a sharp focus. The consequence of this high number of subapertures is high dynamic range images. In addition, as it uses only a combination of vacuum and opaque material, this focussing method is potentially efficient over a very broad wavelength domain. The focal length of such diffractive focussing devices is wavelength dependent. However, this can be corrected. We have tested optically the efficiency of the chromatism correction on artificial sources $(500<\lambda<750 \mathrm{~nm})$ : the images are diffraction limited, and the dynamic range measured on an artificial double source reaches $6.210^{-6}$. We have also validated numerical simulation algorithms for larger Fresnel interferometric arrays. These simulations yield a dynamic range (rejection factor) close to $10^{-8}$ for arrays such as the $3.6 \mathrm{~m}$ one we propose. A dynamic range of $10^{-8}$ allows detection of objects at contrasts as high as than $10^{-9}$ in most of the field. The astrophysical applications cover many objects in the IR, visible an UV domains. Examples are presented, taking advantage of the high angular resolution and dynamic range capabilities of this concept.

Keywords Diffractive focussing $\cdot$ Formation-flying Exoplanet detection

\section{Introduction}

The Fresnel Interferometric imager is based on the Fresnel zone plate concept [38]. Several proposals involving Fresnel zone plates in space have been made since 1993: [6, 10, 19], and [28]. Diffractive optics are very chromatic, and without a way to correct that chromaticism, they would allow observations only in a narrow bandpass.

The chromaticity issue has been addressed by Hyde [19] and Chesnokov [6], with a solution previously proposed and optically validated by Faklis and Morris [11] using the optical principle by Schupmann [34] of correcting optical defects in a conjugate plane. Broadband, chromatically corrected imaging is now achieved with diff ractive optics.

The correction scheme in space is the following: a "secondary module" is placed at prime (uncorrected) focus, a few kilometers downstream the primary array. This module holds a "field telescope", which has a diameter ten times smaller than that of the primary array, e.g. $0.5 \mathrm{~m}$ for a $5 \mathrm{~m}$ array. Close to this telescope and in the same module is a small diffracive Fresnel lens that corrects chromaticism. This corrector element can be made very small: $10 \mathrm{~cm}$ in diameter. It can be a high efficiency blazed Fresnel grating, transmissive or reflective. 
The optical concept of the mission we propose, while being based on diffractive focussing too, differs from the previous ones in several aspects. We have proposed [25]:

- an interferometric approach,

- orthogonal geometry,

- the use of vacuum instead of optically active media,

- high dynamic range applications.

This Fresnel Interferometric Imager proposed (abbreviated "FII" in the rest of the paper) is a demonstrator mission. The $3.6 \times 3.6 \mathrm{~m}$ size has been chosen to fit unfolded as a rocket payload. Our rationale is to favour simplicity, while preserving first order scientific goals. If a space mission proves the efficiency of the Fresnel Interferometer concept, then much larger arrays could be envisioned for later projects.

This Fresnel interferometric imager demonstrator mission being only 3.6 $\mathrm{m}$ in size, is less sensitive than an equivalent size mirror telescope. However, its "niche" will be in the very high quality wavefront provided by diffractive focussing: the manufacturing quality required for a Fresnel array is in the order of millimeters for a $\lambda / 50$ wavefront, and contrarily to standard optics, for a given manufacturing precision the resulting wavefront quality is independent on the wavelength.

Due to the high contrast capabilities, the FII demonstrator shares with TPFC or TPF-I, or the Darwin project, an exoplanet detection capability (though at shorter baselines and shorter wavelengths), but is less specialized for that goal.

Fig. 1 Circular (Soret) zone plate and example of orthogonal Fresnel array, 15 Fresnel zones (half sides)
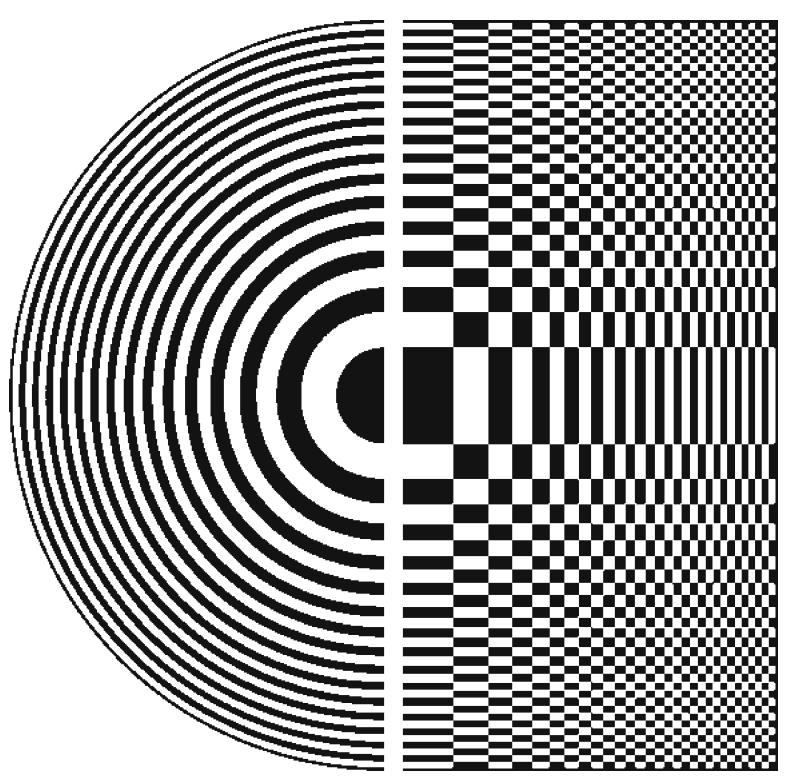
Fig. 2 Image of a point source (point spread function computed by Fresnel transform). Left pupil plane; right image plane

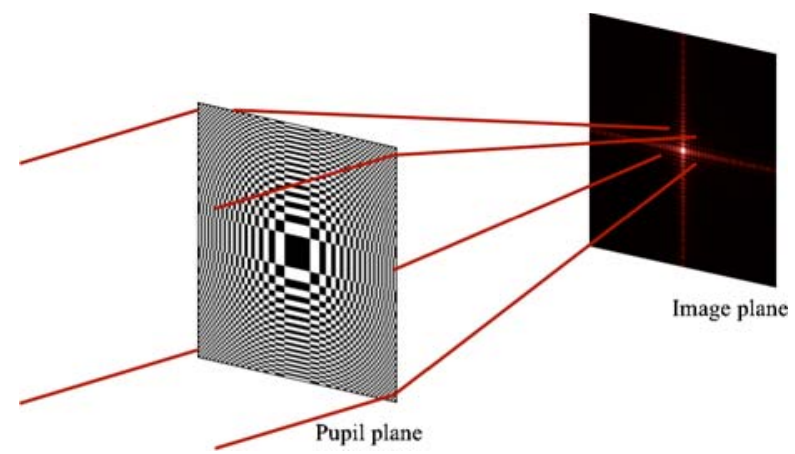

This main focussing element of the FII is a Fresnel interferometric Array (abbreviated FIA). The FIA plays a role equivalent to the primary mirror in an astronomical telescope. The FIA can be seen either as an aperture synthesis array or as a particular case of "Fresnel" zone plate. As are the rings in a zone plate, the subapertures in the FIA are positioned so that a $2 \pi$ phase shift occurs between neighboring apertures, but contrarily to a binary zone plate, the opaque zones of an FIA are connex and create an orthogonal checkerboard-like layout, allowing the use of vacuum for the transmissive zones (subapertures) while preserving mechanical cohesion of the whole frame. Still, same as in a zone plate, the subaperture positioning law turns an incoming plane-wave into a spherical outgoing wavefront, converging into a focus.

From the "interferometric array" point of view, one can see the subapertures as elements of an aperture synthesis array, each casting a beam. The numerous beams from the individual subapertures are coherently combined into focus, without the need for any kind of optical element at the subapertures level, nor any beam combiner downstream. An image is directly formed at focus, the high number (millions) of beams leading to a compact and highly contrasted point spread function (PSF) (Figs. 1 and 2).

\section{Advantages of the Fresnel arrays}

As for an interferometric array, the angular resolution of an FIA is the same as that of a solid aperture having the size of the whole array.

The use of vacuum for the individual subapertures eliminates phase defects and spectral limitations, which would result from the use of a transparent or reflective material. The spectral span of FIAs is bounded by the characteristics of the opaque foil: towards the UV by its effective opacity, towards the IR by its thermal emission. A spectral domain spanning from $100 \mathrm{~nm}$ to $10 \mu \mathrm{m}$ is expected, depending on the nature of the foil and the temperature at which it will stabilize in space. 
The resulting wavefront quality is restricted by the precision to which subapertures are carved. For the long $F$-ratios considered here, this constraint is very loose compared to optical surfacing: a $\lambda / 50$ quality wavefront (required for high dynamic range imaging) is obtained either with a $\lambda / 100$ mirror $(5 \mathrm{~nm}$ precision for the visible), or with a $0.1 \mathrm{~mm}$ precision subaperture positioning in the plane of the FIA. The tolerance perpendicularly to the aperture plane is in the order of centimeters. The positioning constraints in a FIA are not wavelength dependant.

The pattern of the array: all subaperture edges following two orthogonal (or close to orthogonal) directions, casts light at focus into a central peak flanked by two thin orthogonal spikes, confining the light that would be spread into the diffraction rings of a circular aperture, or into the broad side lobes of a diluted interferometric array. Most of the image field of a FIA is thus at very high contrast ratios. To further increase the dynamic range, apodization can be used, either by modulating the size of subapertures from center to limb of the array, or else at a pupil plane after focus, with "Phase Induced Amplitude Apodization" dedicated optics [15].

\section{Drawbacks of Fresnel arrays}

A FIA focusses only part of the light. An incoming plane wave is turned into an outgoing spherical wave, but "turned" is not the right term, one should rather say "split". The FIA acts as a diffraction grating, the converging wave being its "order 1" and the residual emerging plane wave its "order 0 ".

Half of the incoming light is blocked by the opaque foil, then a major part of the transmitted light remains a plane wave, so only $5 \%$ to $10 \%$ of the initial light is focused, this proportion depending on the subapertures shapes and positioning. As a consequence, a three- to fourfold increase in diameter is required to collect the same number of photons as with a solid aperture. The comparison in terms of image brightness depends if the object is extended or unresolved. If comparison with filled apertures is made in terms of cost rather than size, per photon or per valuable datum, FIAs should still get the advantage.

Strong chromatism occurs as a consequence of diffractive focussing at order 1: $f=C_{g r}^{2} / 8 N \lambda . f$ is the focal, $C_{g r}$ the side dimension of the array, $\mathrm{N}$ the number of Fresnel zones within the array (the number of $2 \pi$ phase shifts from center to limb. This chromatism is cancelled by a small diffractive element in the focal instrumentation, operating at diffraction order -1 . This secondary diff ractive element is reflective or refractive, and blazed to optimize transmission at close to $100 \%$. The chromatic correction being made by a dispersion equal and opposite to the initial dispersion, the resulting beam is completely achromatic.

However, the correction occurs after the prime focus, and although the size of the chromatic corrector (adjusted to the pupil size) can be made small, 


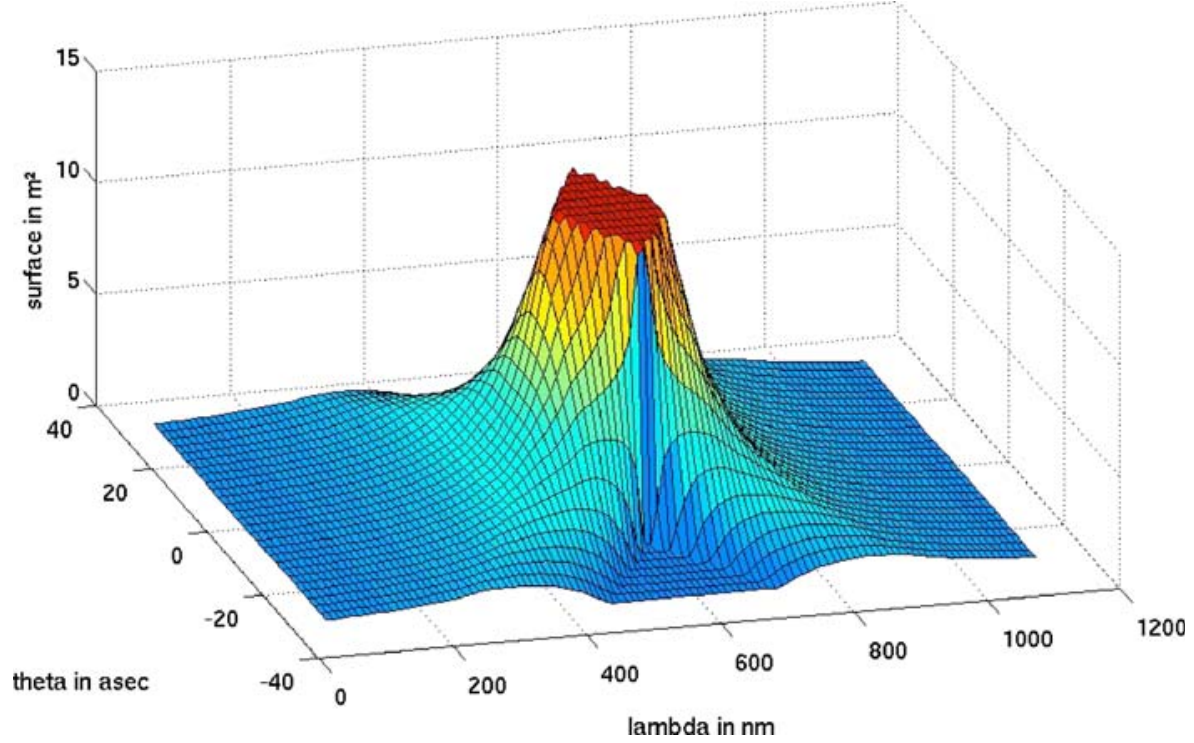

Fig. 3 Collecting area of the main array as a function of the wavelength and angle in the field. The plateau corresponds to the full aperture (unvignetted), the side-lobes correspond to the decrease in beam section due to vignetting by the edges of the field optics when an off-limit wavelength or field angle is reached

the beam entering the first focal plane is grossly unfocussed at wavelengths unadapted to the inter-spacecraft distance.

So, for a given flight configuration, the spectral bandpass is limited to $\Delta \lambda / \lambda=1,41 D / C_{g r}$ for a zero unvignetted field, and less for large fields, $D$ being the diameter of the field telescope (e.g. $68 \mathrm{~cm}$ ) and $C_{g r}$ the side of the square FIA (e.g. $3.6 \mathrm{~m}$ ). The field optics in the secondary spacecraft cause vignetting at wider bands, limiting to $\Delta \lambda / \lambda \simeq 20 \%$ in the above example (Fig. 3).

With several adjustable wavelength channels side by side, we propose to cover sequentially regions of interest in the UV, visible and IR domains. Light will be fed into one channel at a time, and the inter-spacecraft distance adjusted accordingly.

Long focal lengths are implied. For example 2.5 to $18 \mathrm{~km}$ for a $3.6-\mathrm{m}$ array operated at respectively $\lambda \simeq 1 \mu \mathrm{m}$ and $\lambda \simeq 100 \mathrm{~nm}$, therefore requiring formation flying in a low gravity gradient region of space.

\section{Breadboard design and optical tests}

In order to test the concept before presenting a proposal for space, we have built a 22-m focal length prototype and tested it at Observatoire Midi-Pyrénées (Toulouse, France) [33]. The main diffractive focussing array of this prototype 
Fig. 4 Eighty millimeters square Fresnel primary array carved into a thin $80 \mu \mathrm{m}$ stainless steel foil, used for optical tests (58 Fresnel zones, 26,680 subapertures)

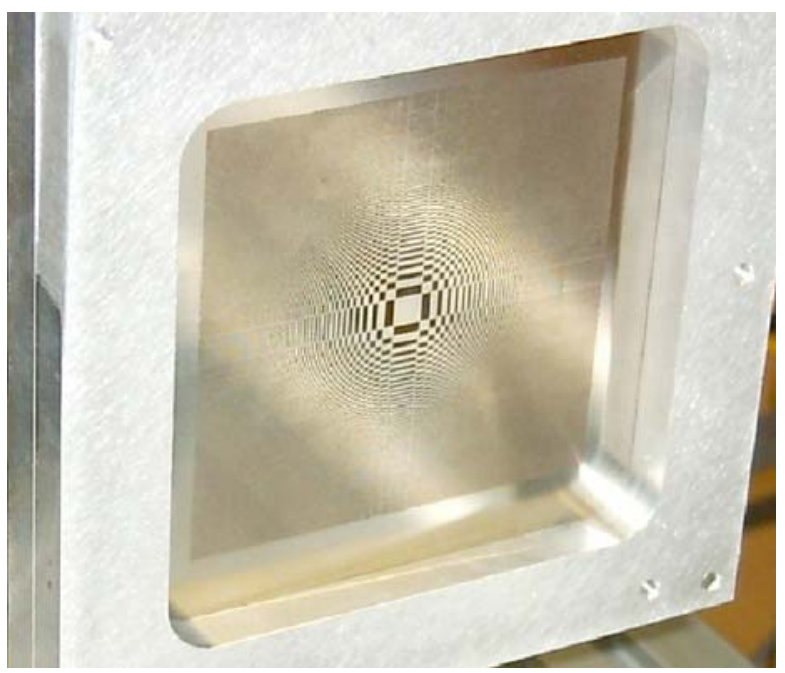

is only an $8 \mathrm{~cm}$ square, punched with 26,680 miniature rectangular subapertures: see Fig. 4. We have tested the PSF quality of the prototype and the efficiency of the chromatism correction on artificial sources $(500<\lambda<750$ $\mathrm{nm}$ ), the image quality (diffraction limited) and the dynamic range. At present, the highest dynamic range of a detected double source is $6.210^{-6}$ with this optical prototype.

We have used the tools developed for the testbed simulation and qualification, to produce numerical simulations for larger Fresnel interferometric arrays. These simulations yield a dynamic range (rejection factor) close to $10^{-8}$, the dynamic range being defined as the ratio of: the average intensity in the image field outside the central lobe of the point spread function (PSF) and its spikes, over the maximum intensity in central lobe of the PSF.

A dynamic range of $10^{-8}$ allows detection of objects at higher contrasts in the whole field except in the spikes, as the limiting factor is the variance of the noise, not the background noise itself, which can be subtracted in the recorded images using a reference source. In a recent publication [25] we present simulations of exoplanet detections with contrasts of $10^{-9}$. A coronagraphic focal instrumentation will further improve this dynamic range: see Figs. 5 and 6 .

\section{Space mission proposed}

The "class L" mission we have proposed is a two spacecraft Formation-Flying telescope, the "Fresnel Interferometric Imager" (hereafter abbreviated FII) orbiting the L2 Sun-Earth Lagrangian point. It addresses different themes of the Cosmic Vision plan, within the guideline questions: "What are the 


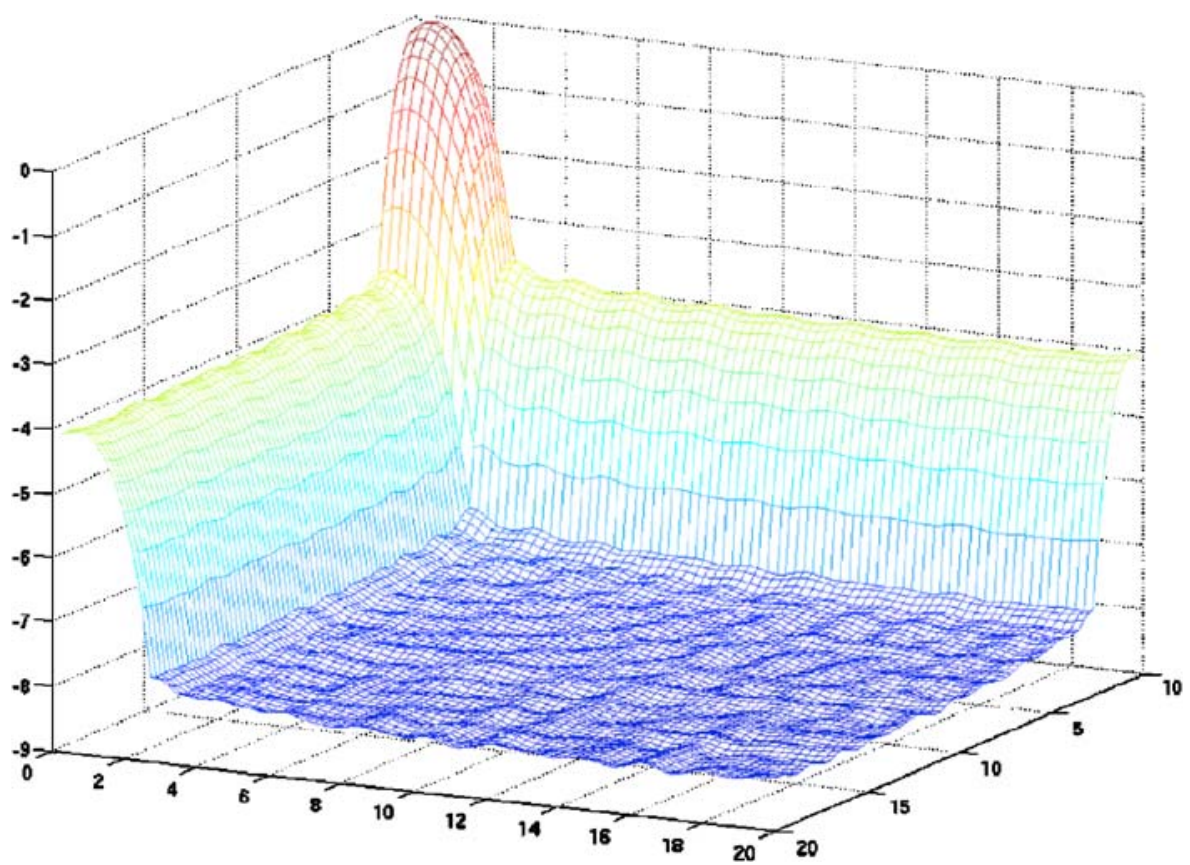

Fig. 5 Simulated quarter field PSF of an apodized 300 zone Fresnel array. Vertical axis log normalized brightness. Horizontal plane position in the field, in units of diffraction limited resolution radius (resel)

Fig. 6 Numerical simulation of the efficiency of a blazed Fresnel chromatic correstor at order -1 versus wavelength (in micrometers). The two top curves are quasi superimposed. They correspond to a non digitized blaze profile and a 32 steps blaze profile. The dispersion corrector lens in our prototype Fresnel imager tested has 128 levels

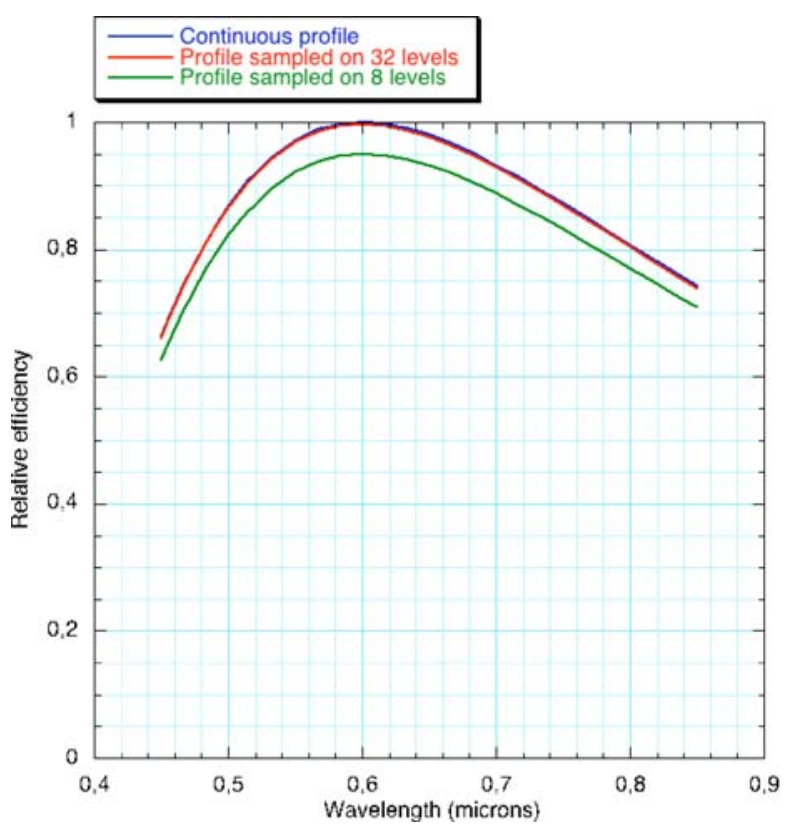


conditions for planet formation and emergence of life?" and "How did the Universe originate and what is it made of?"

\subsection{Spectral coverage}

The spectral domain of the FIA is very broad: $100 \mathrm{~nm}$ to $10 \mu \mathrm{m}$, restrained at short wavelengths by the light-blocking efficiency of the thin opaque foil, and by the decrease in reflectivity of the field optics mirrors coatings. At long wavelengths, by the Planck emission of the opaque foil.

The FII will cover the spectrum with up to six channels: from Lyman $\alpha$ to close IR with angular resolutions from 7 mas at Lyman $\alpha$, to 57 mas at $1 \mu \mathrm{m}$. These channels provide a $\Delta \lambda / \lambda=0.2$ spectral coverage for a given inter-spacecraft distance. Only one spectral channel at a time will be observed in this two-spacecraft formation-flying configuration, the inter-spacecraft distance determining the central wavelength of the observed spectral band. The central wavelength of each channel will be adjustable to some extent, but high rejection rates and dynamic ranges will only be achieved close to the nominal wavelength of each channel $(\Delta \lambda / \lambda<0.1), \lambda$ for each band being determined by the blaze angle of the corresponding chromatic corrector lens.

The six dedicated focal instrumentation channels will share the same $3.6 \mathrm{~m}$ FIA and the same $60 \mathrm{~cm}$ "field optics" mirror. Downstream, all channels will provide imaging capabilities $(860 \times 860$ resels $)$. This is a small field compared to that of a standard telescope. However, being given the long exposure times planned: a few hours per target, it will be possible to split this exposure time and make an $n \times n$ mosaic ( $n=2$ to 5 for example) of contiguous $860 \times 860$ resels fields, thus enlarging the effective field by a factor of $n / \sqrt{2}$. This would cause a very small increase in eregol expense, due to the necessary field shifts: less than $0.01 \mathrm{~m} / \mathrm{s}$ for a 5 by 5 mosaic (i.e. a 3,040 by 3,040 square field), if a continuous raster scan is made. An acceleration is needed only at the end of each scan line, not for each frame in the mosaic [30].

Two or three channels will have coronagraphic optics and low dispersion spectro-imaging capabilities, the others will have a high dispersion spectro fed from the center pixels of image the field. For each channel there will be two detectors, one for broadband imaging and one for spectral analysis of a local zone of the field.

For an FIA transmission efficiency of $8 \%$, the proposed scientific program of the FII will correspond to that of an equivalent $1.3 \mathrm{~m}$ diameter telescope in terms of collected light and $3.6 \mathrm{~m}$ in terms of angular resolution. For point sources, the brightness per unit angle in the PSF is that of a $2.1 \mathrm{~m}$ diameter aperture: this will be the case for spectral analysis of angularly unresolved sources.

The choice of wavelengths for each band depends on the target missions and will be developed in the following sections.

- In the UV: spectro-imaging at high spectral resolution for exoplanet transits, stellar \& extragalactic objects. 
- In the visible: stellar and exoplanet measurements at moderate spectral resolution.

- In the IR: young stellar objects and planetary systems.

\subsection{Angular resolution}

The 7 to 14 mas angular resolution in the 120 to $240 \mathrm{~nm}$ range is enough for resolving a planet orbiting at 0.07 to $0.14 \mathrm{AU}$ from a $10 \mathrm{pc}$ distant star. The angular resolution would degrade to 0.3 arc seconds at $5 \mu \mathrm{m}$ wavelength, but still allow the resolution of a Jupiter at 3 AU. Once the planet is separated from it's star, direct detection of exoplanetary spectral signatures is possible. The spectral resolution in that case will be limited to 100 , due to the scarcity of exoplanetary light.

\subsection{Field}

The maximum recoverable image field of an interferometric array being dependent on its number of subapertures [24], the very large number of subapertures of an FIA allows a high field/resolution ratios, hence much wider fields than other interferometers. The field of a Fresnel array is limited geometrically by the size of the field optics at the prime focus plane. As the focal lengths are very long, the image scale is large and the field angle $\theta_{\text {field }}$ is more limited than with a monolithic telescope:

$$
\theta_{\text {field }}=8 n \lambda S / C^{2}
$$

where $n$ is the number of Fresnel zones in the main aperture (typically 700), $\lambda$ is the wavelength, $S$ is the diameter of the field optics (typically $60 \mathrm{~cm}$ ), and $C$ the size of the square main array, The resulting field is 17 arc seconds at 120 nm wavelength.

Almost all the field will be at rejection rates superior to $10^{-8}$. Only a small solid angle of zodiacal and exozodiacal lights (the area covered by the PSF and its spikes) are contributing to noise at a given point in the field. Because of the spikes reducing the dynamic range over some of the field, two exposures turned by $45^{\circ}$ will be necessary to cover the whole field at high dynamic range.

\subsection{Dynamic range}

The optical tests made in with $\Delta \lambda / \lambda=0.2$ at the 58 Fresnel zones primary array yield $610^{-6}$, and the numerical simulations for a 350 Fresnel zone array reach $10^{-8}$. A consequent improvement of these values is expected with the implementation (in the optical prototype and in the numerical simulations) of a coronagraphic system. The dynamic range decreases for extended objects and dense fields such as galaxies or angularly extended solar system objects, but the angular resolution remains unchanged. As the present laboratory tests 
have been made with a fused silica diffractive lens blazed for $600 \mathrm{~nm}$, further validation tests are required for the UV domain. What will change is the blaze angle, towards lower values. The groove spacing has to be homothetic to the Fresnel zone structure of the primary array, thus is not directly wavelength dependant. The quality of the small diffractive corrector just after the field telescope is of prime importance for dynamic range, as the light not going into its diffraction order -1 may end up polluting the field. Optical tests are planned in the soft UV range for the months to come.

\section{Scientific objectives}

The scientific program presented below covers the "short wavelength" part of a Fresnel imager's observable domain. This choice has been made because of the intrinsic richness of physical phenomena in the UV, and also to take advantage of the larger angular resolutions induced by short wavelengths, allowing competitive resolutions with a $3.6 \mathrm{~m}$ aperture "probatory" mission.

If in the future larger FIAs are envisioned, there will be many targets in the spectrum up to $10 \mu \mathrm{m}$, and sufficient angular resolution to realize for example exoplanet study in the IR.

The fact that the instrument is a formation flying system and that the focal length depends on the wavelength, implies spending ergol for each waveband change, as well as for pointing each new target. The total distance involved depends of the optimization of the observing procedure and target list. A proposed scheme is to observe a given number (e.g. 20) of nearby targets in a given waveband, and optimize a path in order to reduce the total angular distance traveled to cover them all. After this first run completed, the interspacecraft is changed to reach a new waveband, and the procedure is repeated on the same target group. As stated before in this paper, an individual target can be in fact a mosaic of contiguous fields.

Another important factor is the time allowed for reconfiguring the formation: the ergol expense being directly proportional to the total of speed changes required. There is a tradeoff between the total number of targets reachable and the total time required for the array reconfigurations.

During a phase zero study ongoing at CNES, we have developed a few observing schemes, which show that a good compromise is to allow a few hours for target change or focal change, and allow a few hours observing time on each target within a given waveband (Table 1)

For example, with a $500 \mathrm{~m} / \mathrm{s}$ ergol reserve onboard, assuming a $2 \mathrm{~h}$ time required for a focal change or an object shift, and a $0.5^{\circ}$ average separation from one target to the next, 6,500 images can be taken with a two-waveband system in the UV (one centered at $200 \mathrm{~nm}$ and one at $300 \mathrm{~nm}$. If each image is made at a $2 \mathrm{~h}$ exposure time, the total mission duration would be 5.2 years. 
Table 1 This table shows the total number of targets (images) observable for three types of missions, and the associated parameters

\begin{tabular}{|c|c|c|c|c|c|c|c|c|c|c|c|c|c|c|}
\hline Missions examples & $\begin{array}{c}\operatorname{lamb} \\
\mathrm{min} \\
(\mathrm{mu})\end{array}$ & $\begin{array}{l}\operatorname{lamb} \\
\text { max } \\
\text { (mu) }\end{array}$ & $\begin{array}{c}\text { Arraiy } \\
\text { size } \\
(\mathrm{m})\end{array}$ & $\begin{array}{c}\mathrm{Nb} \text { of } \\
\text { Fresnel } \\
\text { zones }\end{array}$ & $\begin{array}{l}\text { Focal } \\
\max \\
(\mathrm{m})\end{array}$ & $\begin{array}{c}\text { Focal } \\
\text { min } \\
(\mathrm{m})\end{array}$ & $\begin{array}{c}\text { target } \\
\text { separation } \\
\text { (degrees) }\end{array}$ & $\begin{array}{c}\text { reconfig } \\
\text { uration } \\
\text { travel } \\
(\mathrm{m})\end{array}$ & $\begin{array}{c}\text { cost of a } \\
\text { target } \\
\text { shift } \\
(\mathrm{m} / \mathrm{s})\end{array}$ & $\begin{array}{c}n b \text { of } \\
\text { consecutive } \\
\text { targets } \\
\text { wiithin a } \\
\text { band }\end{array}$ & $\begin{array}{c}\text { cost of a } \\
\text { spectral } \\
\text { band shift } \\
(\mathrm{m} / \mathrm{s})\end{array}$ & $\begin{array}{c}\text { total } \\
\text { images } \\
\text { taken }\end{array}$ & $\begin{array}{c}\exp \\
\text { time per } \\
\text { image } \\
\text { (s) }\end{array}$ & $\begin{array}{l}\text { mission } \\
\text { duration } \\
\text { (years) }\end{array}$ \\
\hline $\begin{array}{c}\text { Galactic \& extragalactic } \\
\text { in the UV }\end{array}$ & 0.2 & 0.3 & 3.5 & 700 & 10938 & 7292 & 0.5 & 95 & 0.03 & 20 & 1.0 & 6482 & 18000 & 5.2 \\
\hline $\begin{array}{l}\text { Photosphere and } \\
\text { protostellar disks } \\
\text { imaging }\end{array}$ & 0.25 & 0.50 & 3.5 & 700 & 8750 & 4375 & 0.2 & 31 & 0.01 & 20 & 1.2 & 7221 & 3600 & 2.5 \\
\hline $\begin{array}{c}\text { Small solar system } \\
\text { bodies }\end{array}$ & 0.25 & 0.50 & 3.5 & 700 & 8750 & 4375 & 1.0 & 153 & 0.04 & 20 & 1.2 & 4847 & 7200 & 2.2 \\
\hline
\end{tabular}

An average 2-h exposure time is supposed per target, and a 2-h reconfiguration time between targets is assumed

Observing at shorter wavelengths would shorten all the distances and increase the number of reachable targets before exhausting the ergol reserve.

\subsection{Observations in the far UV}

The UV spectral range is particularly difficult to reach below Lyman $\alpha$ because of limited reflectivity at short wavelengths. However, this domain provides a unique tool to observe gas in numerous astrophysical objects since there are many strong electronic transitions, including of most of the atomic species, ions and molecules. The study of exoplanetary atmospheres can be achieved using high dynamic range techniques (transit or direct imaging) Both these subjects fall within the ESA Cosmic Vision AO aimed at either the understanding of the Universe as a whole as well as providing significant steps in the search of other habitable planets. The spectral domain below Lyman $\alpha$, from 912 to 121.6 $\mathrm{nm}$, is particularly known for its instrumental inaccessibility (observed only via COPERNICUS in the 1980s and FUSE between 1999 and 2007) as well as for its remarkable richness in atomic, ion and molecular lines. Many of these lines are of very important astrophysical species ranging from the more abundant atoms including $\mathrm{H}, \mathrm{D}, \mathrm{C}, \mathrm{N}$, and $\mathrm{O}$, to ions in several states, as in particular $\mathrm{CI}$, CII, CIII (and CIV in the UV near $155.5 \mathrm{~nm}$ ), SiI, SiII, SiIII, SiIV, OI and OVI. The UV also contains some of the most important astrophysical molecules as $\mathrm{H}_{2}$, HD and CO. See Fig. 7.

The FUSE observatory was selected by NASA, launched in June 1999, and has been operating for more than 8 years (references [10] to [14]). In spite of the technological difficulties, particularly the poor coating efficiencies below $110 \mathrm{~nm}$, the effective area of the FUSE observatory is still "quite high" because only two reflective surfaces were used in its design. The FUSE effective area is on the order of $50 \mathrm{~cm}^{2}$. In comparison, the Fresnel Imager effective area can be computed as follows:

$$
A=C^{2} t_{\text {primary }} t_{\text {field mirror }}^{2} t_{\text {grating }} Q E_{\text {detector }}
$$

Assuming a collecting area $C^{2}=3.6 \times 3.6 \mathrm{~m}^{2}$, an efficiency $t_{\text {primary }}=8 \%$ (wavelength independent) of the diffractive focussing, a field optics mirror 
Fig. 7 This figure underlines the spectacular rise in number of available diagnostic lines when moving from the visible to the UV and finally to the far UV (Adapted from the FUSE program)

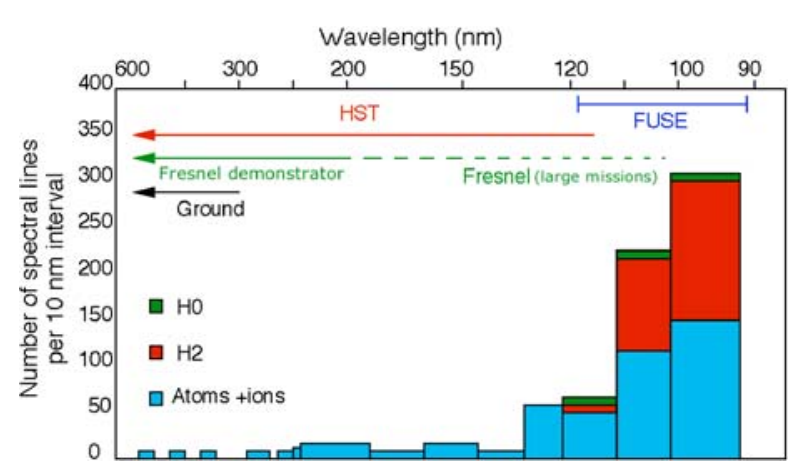

efficiency $t_{\text {field mirror }}^{2}=0.5$, an additional concave Fresnel grating used as chromatic corrector and spectrograph, having a $t_{\text {grating }}=0.6$ efficiency, and a detector efficiency of 0.2 , this yields an effective area $A=622 \mathrm{~cm}^{2}$. This should give access to objects 2.7 magnitudes fainter than those presently reached by $50 \mathrm{~cm}^{2}$ of the FUSE observatory, notwithstanding the gain in angular resolution: 7 milli-arc seconds at Lyman $\alpha$.

To illustrate a few of the possibilities to be opened by such a gain in sensitivity, one could mention [12-14, 29]:

- the study of the intergalactic medium observed with FUSE via a small handful of bright quasars and AGNs down to 14th magnitude. The FII would be opened to almost all known similar objects down to 18th magnitude, i.e. thousands of potential targets;

- the study of the galactic elemental abundance in the ISM [35], presently limited to 13 th magnitude hot stars. This study could be extended to 16 th magnitude providing access not only to the whole Galaxy, but even to nearby ones such as Andromeda;

- the deuterium abundance, both a unique tracer of galactic evolution and of the primordial Universe, could be evaluated much further in the Galaxy and in more than only one extragalactic target as presently done by FUSE;

- The observation of the physical conditions further inwards from the outer layers of the molecular cloud cores, and their interaction with the ambient radiation field (for instance in dense star forming regions like Orion), particularly in $\mathrm{H} 2$ and $\mathrm{CO}$ molecules, will become possible while it is limited for the moment, complementary to the radio observational approach, to the still diffuse envelopes of these molecular clouds; the ionized environment of galaxies, including the cooling flows as traced by the unique OVI lines, could become accessible beyond our own galactic environment to numerous galactic clusters;

- The bright nucleus of the Seyfert galaxy PG 1259+593 has been observed with FUSE. This object of magnitude $\mathrm{V}=15.84$ could be reachable at lower integration times with the Fresnel imager.

- the planetary formation process via the physical state of dense accretion disks, followed by diffuse debris disks, where earth like planets are formed. 
These processes are particularly well analysed in the far UV spectral range where many important diagnostic lines are available; only a few disks are accessible to FUSE observations. With a gain in sensitivity of a factor of 12 compared to FUSE, a Fresnel telescope would allow scrutinizing the most abundant molecules like $\mathrm{H} 2$ and $\mathrm{CO}$ in proto-planetary disks and debris disks much further in the Galaxy and would allow reaching those forming planetary systems in nearby star forming region like in TaurusAuriga and Orion regions. Changing the sensitivity of an observatory in a spectral range by a 12 factor is never a trivial step, but in the specific case of the far UV domain, this gain will open up access to most of the possible targets known, changing our vision of any given problem from the current first few hints, to a complete statistical and detailed survey. The targets available in the UV are, even now, all catalogued by the GALEX mission and thus are known to number in the thousands. Moving our observational possibilities in new astrophysical regions always bring new answers and surprises.

\subsection{Study of the exoplanetary atmospheres and lithospheres}

With the study the exoplanetary atmospheres, three possible approaches could be considered:

- Direct method, trying to observe the light reflected by the planet, and through the spectral analysis deduce the atmospheric and surface composition; this approach requires direct imaging with high angular resolution and rejection rates to properly resolve the planet from the star.

- The favourable cases when the planet passes behind its parent star (often called secondary or anti-transit) allowing an evaluation of the amount of planetary reflected light by difference.

- The favourable cases when a planet transits in front of its parent star. During these moments, the stellar light filtered by the planetary atmosphere reveals signatures of its extent, structure, and composition [37].

These approaches are complementary, one should note interesting advantages of observing transit and direct imaging or secondary transit:

- Direct imaging will add orbital data such as semi-major axes and $\sin (i)$, important by many aspects such as obtaining a model independent planet surface temperature. Orbital information may not be available otherwise if there are no transits and if the induced star motion is beyond the sensitivity of Doppler detection.

- When a given planet transits, it also always anti-transits which gives in all those cases the access to the planetary atmosphere, structure and composition over the sunlit planetary atmosphere (secondary transit) as well as its terminators (primary transits).

- The probability of having a transiting planet at about $1 \mathrm{AU}$ from a star is $0.5 \%$. Under the assumptions of a uniform distribution of planetary 
systems and Earth-like bearing planetary systems in the Solar neighbourhood, if one searches for such planets by direct imaging within $10 \mathrm{pc}$, one should find the same amount of potential targets to be studied via both transit and anti-transit within 60pc from the sun [3-5].

Among the lines that could be searched in the transiting exoplanets spectrum, are (Figs. 8, 9 and 10):

- Hydrogen at 121.6, carbon at $130.5 \mathrm{~nm}$, oxygen at 130.2 and $133.5 \mathrm{~nm}, 0_{3}$ at $300 \mathrm{~nm}, \mathrm{Na}$ and $\mathrm{K}$ at 600 to $900 \mathrm{~nm}$ (hot Jupiters), $0_{3}$ at $800 \mathrm{~nm}$.

- $\mathrm{H}_{2} \mathrm{O}$ at $1.8 \mu \mathrm{m}, \mathrm{CO}_{2}$ at $1.9 \mu \mathrm{m} . \mathrm{NH}_{3}$ between 3 and $4 \mu \mathrm{m}$, CO between 4 and $5 \mu \mathrm{m}$. For direct imaging and spectroscopy, there is need for both angular resolution and high dynamic range in the low part of the light histogram (rejection rate). The spectral windows will be more limited. Furthermore, the reachable spectral resolution for exoplanets with a $3.6 \mathrm{~m}$ FIA is as low as 70 with a $S / N$ ratio ranging from 3 to a few hundred (see following figures), allowing detection of the following broad lines: $0_{2}$ at $760 \mathrm{~nm}$, chlorophyl break at $700 \mathrm{~nm}$ (possibly at different wavelengths depending on the stellar spectrum). Other lines, such as $\mathrm{H}_{2} \mathrm{O}$ at 1.4 and $1.8 \mu \mathrm{m}$, and $\mathrm{CO}_{2}$ lines between 4.2 and $4.5 \mu \mathrm{m}$ could be a motivation to shift the reddest channel further into the IR, but imaging gets harder due to the loss of angular resolution due to wavelength.

In cases 2 and 3 (transit observations: references [16-21, 23, 31, 32], and antitransit observations: references [24-28, 31, 32]) there is no need for high angular resolution. However, there is need for high dynamic range in the bright part of the spectral lines, and high spectral resolution: the total amplitude of the line variation due to a planet transit like HD209458b is in the order of $10^{-6}$, which means a required dynamic range of $10^{-7}$ if we want to have a $S / N$ of 10 in the planet absorption spectrum [37, 39].

The important point is that the transiting and anti-transiting atmospheric planetary studies have already started with both the Hubble and the Spitzer Observatories. In the transit approach, the main Hubble results are the detection of several atmospheric constituents as NaI, HI, OI, CII and $\mathrm{H}^{+}$as well as the demonstration of atmospheric escape states as violent as the one probably suffered by the early Earth and Venus, i.e. hydrodynamic escape involving all species transported away within the hydrogen flow. In the secondary transit studies, mainly with Spitzer results, the planetary thermal sunlit side emission has been detected and the temperature and albedo evaluated, as well as the first hints of emission distribution over the stellar-lit hemisphere using timing when the planets disappears behind the star.

HST-STIS is the best spectrograph used for such planetary studies. The STIS throughput of the different spectrographs ranges from 1 to few percent from the UV to visible and thus corresponds, in the case of a $2.4 \mathrm{~m}$ diameter telescope, to an effective area of the order of 500 to few $1,000 \mathrm{~cm}^{2}$. In the case of the $3.6 \times 3.6 \mathrm{~m}^{2}$ Fresnel Telescope, one could again reach, with tworeflection field optics design and a relatively simple one-reflection grating 


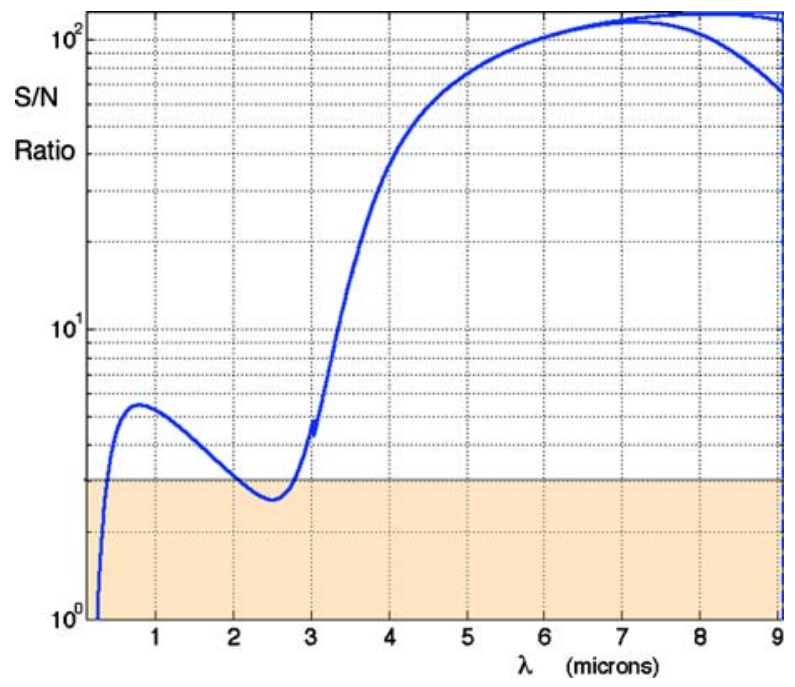

Fig. 8 Signal/noise as a function of $\lambda$ for a "cold" Jupiter at 5.2 AU from a solar type star, $10 \mathrm{pc}$ away. The shaded zone is where $S / N<3$. Zodiacal and Exozodiacal contributions are simulated for a solar type planetary system. Twenty-four hour exposure on a $3.6 \mathrm{~m}$ FIA, rejection rate $210^{-8}$, spectral resolution 70 . The star-planet separation allows sufficient angular resolution up to $9 \mu \mathrm{m}$. IR emission from the planet is observable from 3 to $9 \mu \mathrm{m}$. The small glitch in the curve at $3 \mu \mathrm{m}$ is due to a wavelength dependant change in rejection rate. The bifurcation in the $S / N$ curve at $7 \mu \mathrm{m}$ corresponds to two different temperatures of the opaque Fresnel foil: $70 \mathrm{~K}$ (top) and $80 \mathrm{~K}$ (bottom)

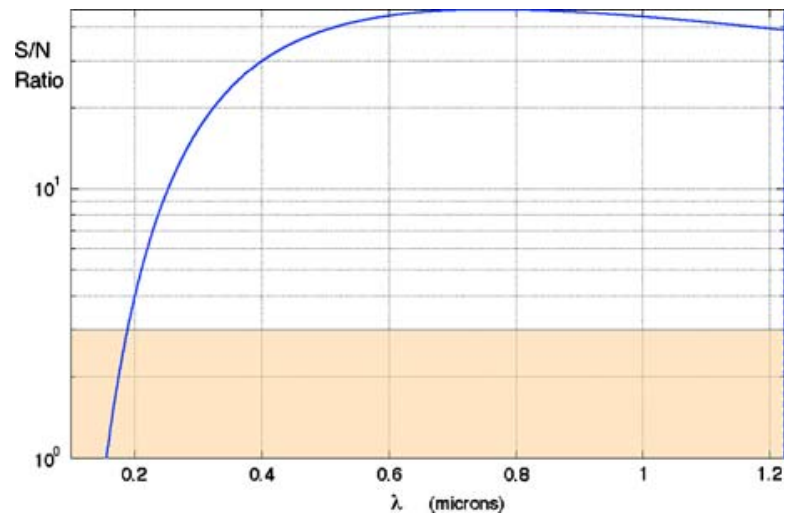

Fig. 9 Signal over noise ratio as a function of wavelength for a "warm" Jupiter at 1 AU from a solar type star, $10 \mathrm{pc}$ away. The shaded zone is where $S / N<3$. One hour exposure time on a $3.6 \mathrm{~m}$ Fresnel array, rejection rate $210^{-8}$, spectral resolution 70 . The fall in $S / N$ ratio below $230 \mathrm{~nm}$ is due to the decrease in number of photons from the solar type spectrum. At the other end of the observable spectrum, the angular resolution limits the wavelength to $1.6 \mu \mathrm{m}$. The shaded zone is for $S / N<3$ 


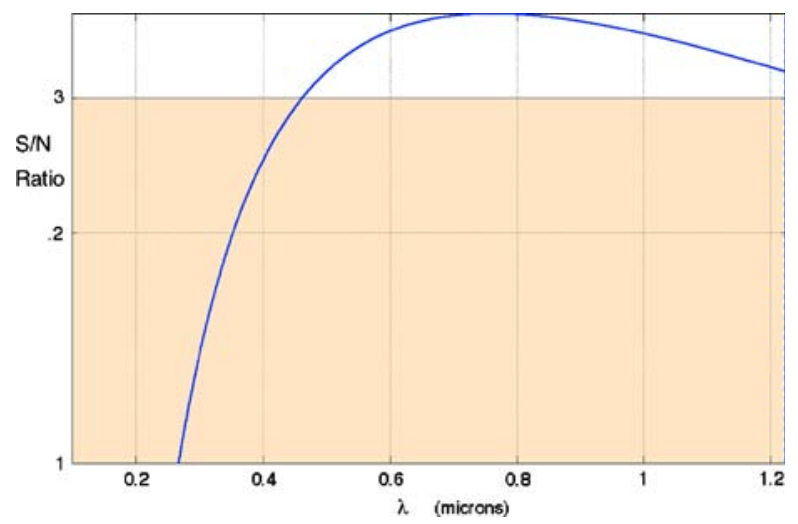

Fig. 10 Signal over noise ratio as a function of wavelength for a "cold" Jupiter at 5.2 AU from a solar "marginally reachable" Signal over Noise ratio as a function of wavelength for a Venus at 0.7 AU from a solar type star, 10 pc away. The shaded zone is where $S / N<3$. One hundred hour exposure time on a $3.6 \mathrm{~m}$ Fresnel array, rejection rate $210-8$, spectral resolution 70 . The angular resolution limits the wavelength to $1.2 \mu \mathrm{m}$. The shaded zone is for $S / N<3$. An Earth at $10 \mathrm{pc}$ is beyond the reach of a $3.6 \mathrm{~m}$ array. It would be marginally reachable, either with a $10 \mathrm{~m}$ array at rejection rate $210^{-8}$, or with the $3.6 \mathrm{~m}$ array at rejection rate $210^{-10}$

design, and a $20 \%$ efficiency detector, nearly a 2,000 to $3,000 \mathrm{~cm}^{2}$ effective area system, depending upon the spectral range considered. Here a gain by a factor of three relative to present observations will allow the same studies accomplished now over eighth magnitude stars to be done in the case of more than 9th magnitude similar objects. It is clear that this will not be enough to reach for potential Earths but will increase the number of atmosphere studied by nearly a factor of 6 , i.e. statistical studies could really begin.

This study being made with a $3.6 \mathrm{~m} \mathrm{FII}$, one can hope that if the first mission is successful, further developments of that design will allow to really reach Earth type planetary atmosphere, using 15 to $30 \mathrm{~m}$ FIAs. See as a prediction of the possibilities in Table 2, taken from the Lecavelier des Etangs and Ehrenreich [12] Cosmic Vision proposal [8].

\subsection{Study of stellar systems, photospheres and close environments}

Since 1990s the Hubble Space Telescope (HST) has been providing highangular resolution views of the Universe from UV to IR wavelengths with an angular resolution close to 0.1 arc second. The diffraction limit of this $2.5 \mathrm{~m}$ telescope is comparable to the ground-based telescopes built over 50 years ago (e.g. Mt. Wilson $2.54 \mathrm{~m}$ telescope). However, the fact that the HST is in space and therefore free of the atmospheric seeing effects, with the crucial capability of observing at UV wavelengths, provided unprecedented opportunities for high-resolution studies of many astronomical sources. For example, HST observations of the symbiotic system Mira AB (at $130 \mathrm{pc}$ ) demonstrate the power of sub-arcsecond resolution by having separated this 
Table 2 Table from Lecavelier des Etangs and Ehrenreich [12]

\begin{tabular}{lcccr} 
Planet & \multicolumn{3}{c}{ Telescope $\emptyset(\mathrm{m})$} & Number of targets \\
for $\mathrm{V}=8, \mathrm{~S} / \mathrm{N}=5$ & $(\mathrm{~S} / \mathrm{N} \geq 5, \emptyset=10 \mathrm{~m})$ \\
& K-star & G-star & F-star & $(\mathrm{N} / \beta \gamma)$ \\
\hline Earth & 15 & 22 & 29 & 14 \\
Venus & 50 & 83 & 113 & $<1(0.3)$ \\
Ocean-Planet & 2.3 & 4.4 & 6.8 & 3400 \\
small Earth & 9 & 13 & 17 & 61 \\
small Venus & 36 & 64 & 92 & $<1(0.9)$ \\
small Ocean-Planet & 1.4 & 2.7 & 4.0 & 13900 \\
super-Earth & 65 & 113 & 142 & $<1(0.1)$ \\
super-Ocean-Planet & 9 & 18 & 29 & 54 \\
\hline Hot Jupiter & 2.0 & 3.5 & 5.5 & 40000 \\
Hot Neptune & 9.0 & 17 & 25 & 440 \\
\hline Evaporating ocean & $<1$ & $<1$ & & 1150 \\
& & & & with $\emptyset=1 \mathrm{~m}$ \\
Evaporating ocean & & & & in Local Bubble \\
\hline
\end{tabular}

Shows the number of planetary targets accessible with an $\sim 10 \mathrm{~m}$ diameter telescope. Although a remote possibility in the case of a classical $10 \mathrm{~m}$ space telescope, a $30 \mathrm{~m}$ FIA having the same collecting power and triple angular resolution may be a simpler solution. Using the approach of Ehrenreich et al. [9] we have adapted the evaluation of detectable planets to a $30 \mathrm{~m}$ FIA as a follow up of our program. We included all varieties of known extra-solar planets: from Earth sized to Jupiter-like, adding their probable satellites, as well as possible ocean planets and early Venus or evaporating ocean type planets. This table demonstrates the huge potential capabilities of our design, which will still shows high probability detections with the proposed $3.6 \mathrm{~m}$ in the easiest cases since at the time this program flies, more transiting planets will be known thanks to the present and/or future ground based (e.g. OGLE, TrES, XO, HAT, etc...) and/or space borne programs (e.g. CoRot and Kepler, etc.)

0.6 " interacting binary for the first time in UV, where the most important signatures of accretion occur [20] Mira $A B$ is the nearest symbiotic system composed of an evolved mass-loosing AGB star (Mira A) and a wind-accreting white dwarf. HST and Chandra images of Mira AB showed evidence for possible direct interaction between Mira A and B, showing that Mira B is accreting not only from the wind of the AGB star, but also via direct mass exchange [22]. This was an unexpected result, because the components are separated by $70 \mathrm{AU}$ and it has been assumed in the past that the interaction between the components in such a system can be carried out only via wind accretion.

Unfortunately, very few interacting binaries have been resolved by direct imaging. Currently, most of our accretion paradigms are based on timeresolved spectroscopic observations. An order of magnitude higher (10 mas) spatial resolution in the UV and optical will offer unprecedented opportunities for detailed studies of nearby stars and interacting binary systems.

The FII will be able to image the Mira AB system with 100 pixels covering the region between the components, and 100 pixels covering the atmosphere of Mira A. Furthermore, the FII will be able to separate the components of several nearby currently unresolved interacting binaries, including symbiotic systems (e.g. CH Cyg, R Aqr, and others). Symbiotics are some of the most fascinating interacting systems because of their dramatic transformations 
and extremely complex circumbinary environment. These systems are very important because they are likely progenitors of bipolar planetary nebulae. They have also been invoked as potential progenitors of at least a fraction of supernovae type Ia, a key cosmological distance indicator [7].

The FII should resolve outflows and circumbinary environments in a variety of systems, including regions of colliding winds. The results will have important implications for our understanding of accretion processes in detached binaries and other accreting systems. Understanding accretion driven flows in binaries will directly affect our understanding of similar flows around YSOs, including planet formation in circumstellar disks.

HST and ground-based observations detected significant asymmetries in the atmospheres of several nearby giants and supergiants, including Mira A and $\alpha$ Ori. However, the causes of these asymmetries remain a mystery. Possibilities range from giant stellar spots, convective cells, to effects of non-spherical pulsation and massive outflows. Resolution of an order of a magnitude higher than that of the HST in the UV is needed to determine the causes of asymmetries observed in these evolved stars. The FII will be able to image the atmospheres and the circumstellar environment of dozens of nearby Mira-type stars and supergiants with approximately 100 pixels over the stellar surface, compared to only few that we currently get using the HST. This will allow determining the causes of the asymmetries and also a detailed study of the structure of the extended atmospheres.

\subsection{Study extra-solar protoplanetary disks: the disk evolution watcher (DEW)}

Observations of extra-solar protoplanetary disks allow understanding the physical evolution and chemical composition of disks at epochs preceding and contemporaneous with the formation of planets. Chemical abundances are determined by physical conditions such as density, temperature and the incident radiation field. The UV radiation field plays a fundamental role in the chemical evolution of the disks:

1. FUV continuum emission induces photodissociation of $\mathrm{CO}$ enhancing organic species such as $\mathrm{CN}, \mathrm{HCN}$ and $\mathrm{HCO}+[1,2]$.

2. Lyman alpha carries about $85 \%$ of the far UV energy flux (see TW Hya observations by Herczeg et al. [18]). Thus the radiation field is selectively dissociating species like $\mathrm{HCN}$ or $\mathrm{H} 2 \mathrm{O}$ while others such as $\mathrm{CO}$ or $\mathrm{H} 2$ are left unaffected [36].

3. The vertical structure of the disk is controlled by the UV radiation of the central source. The disk can be divided into three layers: the upper photodissociation region (PDR), the warm molecular layer below and the midplane cool layer. Low molecular abundances are caused by depletion in the mid-plane.

4. The structure of the inner disk $(r<2 \mathrm{AU})$ is controlled by the evolution of the accretion-outflow engine. The engine transforms gravitational energy into mechanical energy (the outflow) and radiation. The presence of 
magnetic fields makes the dissipative processes to occur at high energies (UV, X-ray radiation) compared with the temperature of the disk and the stellar photosphere. This radiation, in turn, controls disk evolution (see [33], for a recent review).

The FII has the following properties that make it an ideal instrument to follow the evolution from disks to planets:

1. The high dynamical range allows to map simultaneously the illuminating radiation field and its propagation/diffusion in the circumstellar environment. A $10^{7}$ dynamical range makes feasible to track the radiation field to a distance $3.210^{3}$ times the radius of the illuminating source. Assuming this to be the central engine (with a characteristic radius of 10 stellar radii or $0.05 \mathrm{AU}$ ), the FII will map with unprecedented sensitivity and resolution the inner $100 \mathrm{AU}$ with a 7 mas resolution $(0.98 \mathrm{AU}$ at $140 \mathrm{pc}$, the distance to the Taurus or Lupus nests of Pre-Main Sequence stars). This scale reduces to $0.098 \mathrm{AU}$ for AB Dor (a nearby Weak-Line T Tauri star) or to 0.392 AU to TW Hya. For this objective, possible problems caused by zodiacal light will have to be investigated (Taurus is in the ecliptic).

2. The high dynamic range and resolution allows to map the inner region jet with unprecedented resolution and measure the role that the jet may have in the illumination of the inner region of the disk. The simultaneous observation of the jet and the engine will allow to track the correlation between engine and jet variability and derive parameters such as the knots excitation and jet collimation mechanisms.

3. To obtain simultaneously spectra and high resolution images of the central source allows to measure the relative contribution of various lines to the illuminating flux: Lyman $\alpha$, OI, CII and CIV for the Far UV, and provide data on the selective absorption role on the chemical evolution of the disk.

4. The possibility to observe in several spectral windows, extending towards the IR, will allow to map the effect of the irradiation field on the disk atmospheric structure (the warm molecular layer) where $\mathrm{CO}, \mathrm{CO}_{2}, \mathrm{H}_{2} \mathrm{O}$, $\mathrm{NH}_{3}$ emission is observed. Also the role of the UV radiation field in $\mathrm{PAH}$ formation could be studied since some of the most prominent PAHs molecular bands are within the range of the FII. FII will also provide direct information on the extinction law (from $100 \mathrm{~nm}$ to $10 \mu \mathrm{m}$ ) and the properties of the dust lifted from the disk at different locations providing crucial information for future projects like ALMA.

\subsection{Extra-galactic program with the Fresnel interferometric imager}

Several extragalactic projects could be addressed with the FII. These projects take advantage of the capabilities of FII in terms of spatial resolution, wide wavelength domain and high dynamical range as compared to other ground-based and space facilities available or planned beyond 2012-2015. 
FII is intended to start operations after JWST, ALMA and possibly SKA. Therefore, extragalactic science with FII has to be optimized to take maximum benefit from synergy and complementarity with these ongoing projects. FII is expected to substantially contribute to a new panchromatic view of galaxies at all redshifts, thus providing new clues to understand galaxy formation and evolution. This is a non-exhaustive list of extragalactic projects which could be addressed with FIIs:

Evolution of the star formation activity in the universe: a complete census from high- $z$ to the local universe:

Thanks to its UV to near-IR imaging capabilities with optimal spatial resolution, FII is an ideal instrument to study the evolution of star formation activity, traced by the restframe UV flux, from the early epochs to the presentday universe. Indeed, JWST is an infrared-optimized space telescope, hence the UV capabilities of FII are particularly well suited to determine the energy census of galaxies at all redshifts, in particular from the mid- $z(z<2-3)$ to the local universe. The expected impact of FII in this area could be easily evaluated by extrapolating the recent results of GALEX, the only facility presently available (and foreseen) in the UV. FII is expected to complement and to extend the capabilities of GALEX. Because of its small FOV and high spatial resolution (typically one galaxy per shot), FII is intended to be a follow up rather than a survey instrument. FII could be efficiently used to target galaxies selected from utra-deep photometric and spectroscopic surveys (optical + near IR).

Mapping the Star Formation of galaxies in the local Universe:

The high spatial resolution of FII in the UV to near-IR could be used to obtain a detailed mapping of the star-formation activity in the local Universe, both in imaging and spectroscopic modes. An unprecedented resolution could be achieved in the study of the physical properties of star-forming regions by combining the spectroscopic information provided by the ISM and stars in these regions.

\section{Exploring the AGN-starburst connexion.}

The relationship between AGN and starburst activity in galaxies is a very important topic, closely related to the process of galaxy assembly across the cosmic time. Starburst (or post-starburst) activity is usually located around the central AGN. FII could provide a new insight into this subject thanks to its high spatial resolution at all wavelengths from UV to near-IR, and to its very high dynamic range.

Observing the first galaxies with FII: Constraining the physical properties of galaxies at $z>7$

This project is focused on the spectroscopic study of a sample of "bright" $z \sim 7-12$ galaxies, for which we could take advantage of the spatial resolution provided by FII in the near-IR. This is also a test case for an improved 
version of FII with a larger aperture, which could sustaintably improve the capabilities of JWST for the study of the first galaxies in the Universe. Indeed, most $z \sim 7-12$ galaxies are expected to have an angular size below 0.1". The physical properties of such distant galaxies could be derived from Lyman alpha emission, combined with multi-wavelength photometry. Two different cases could be explored in this prospective phase: High $-z$ galaxies selected around the critical lines in mid- $z$ strong-lensing clusters. In this case, the FOV of FII in the near-IR is ideally suited for this study. The detection of magnitude-limited samples of $z>7$ galaxies is a factor between a few and a few ten times more efficient in lensing clusters than in blank fields (depending on FOV -especially for a FOV of $\sim 1-2$ arc sec, redshift of lenses and sources, LF of sources...), and the relative efficiency increases with the redshift of the sources. The survey could be either "blind" (because the position of the critical lines at such high$z$ are known with well suited accuracy), or "pointed" after the photometric pre-selection of candidates found in previous (ultra)deep surveys of lensing clusters. The later is better suited for the prospective phase.

High- $z$ galaxies selected in blank fields from previous studies. Utra-deep optical+near-IR surveys (broad-band filters) are presently ongoing or planned, using different wide-field IR cameras. The new generation of ground-based near-IR spectrographs should be able to confirm the most promising "bright" candidates. However, the combined gain of FII in spatial resolution and ideal spectral resolution could be crucial to confirm a fraction of the fainter candidates, as well as a privileged test-case for the next version of this telescope.

\section{Conclusion}

The Fresnel imager can be considered as an "aperture multiplier": it uses a modest dimension two-reflection solid aperture telescope as field optics, onto which light is focussed form upstream, by a diffractive aperture array five to ten times larger in diameter. This Fresnel array provides the angular resolution, imaging capabilities and wavelength independent focussing efficiency (independent in terms of transmission, not focal length) of a large aperture, without the manufacturing and weight constraints of building and launching a large mirror. Future space telescopes of this type using 50 to $100 \mathrm{~m}$ foils could lead to sub milli arc-seconds resolutions with two-vessel formation flying systems having 4 to $400 \mathrm{~km}$ focal lengths. These future missions could be an alternative to large solid aperture space telescopes or multi-telescope space interferometers.

In case of large arrays, formation flying with more than two spacecrafts would allow simultaneous observations at two or more spectral bandpasses, each spacecraft being at its corresponding focal length along the optical axis. Central obscuration would occur with the present centred Fresnel design for the last-in-row receptors.

In addition to being a possible precursor of very large lightweight apertures in space, the strength of Fresnel imager proposed here is also in its dynamic 
range and capability to operate over a variable spectral range. This unique possibility could open two very important astrophysical research domains in which members of our group have considerable expertise and significant discovery results: observations in the far UV and the study of exoplanetary atmospheres.

\section{References}

1. Aikawa \& Herbst.: A\&A, 351, 233-246. http://adsabs.harvard.edu/full/1999A\&A...351...233A. (1999)

2. Aikawa \& Herbst.: A\&A, 371(3), 1107-1117 (2001)

3. Ballester, G.E., Sing, D.K., Herbert, F.: The signature of hot hydrogen in the atmosphere of the extrasolar planet HD 209458b. Nature 445, 511-514 (2007)

4. Charbonneau, D., et al.: Detection of an extrasolar planet atmosphere. Astrophys. J. 568, 377384 (2002)

5. Charbonneau, D., et al.: Detection of thermal emission from an extrasolar planet. Astrophys. J. 626, 523-529 (2005)

6. Chesnokov, Y.M.: A space-based very-high resolution telescope. Russ. Space Bull. 1(2), (1993)

7. Chugai, N.N., Yungelson, L.R.: Astron. Lett. 30, 65 (2004)

8. Deming, D., et al.: Infrared radiation from an extrasolar planet. Nature 434, 740-743 (2005)

9. Ehrenreich, D., et al.: The transmission spectrum of Earth-size transiting planets. Astron. Astrophys. 448, 379-393 (2006)

10. Early, J.T.: Solar sail-Fresnel zone plate lens for a large aperture based telescope. AIAA 1705, 3773-3778 (2002)

11. Faklis, D., Michael Morris, G.: Broadband imaging with holographic lenses. Opt. Eng. 28(6), 592-598, June (1989)

12. Lecavelier Des Etangs, A., Ehrenreich, D.: A large UV-optical telescope for characterization of the atmospheres of extrasolar planets and satellites. In: Favata, F., Sanz-Forcada, J., Gimènez, A., Battrick, B. (eds.) 39TH ESLAB Symposium on Trends in Space Science and Cosmic Vision 2020, held 19-21 April 2005, Noordwijk, The Netherlands. ESA SP-588. European Space Agency, 73L (2005)

13. de Castro, G.A.I., Lecavelier, A., D’Avillez, M., Linsky, J.L., Cernicharo, J.: APSS 303, 33-52 (2006)

14. Grillmair, C.J., et al.: A spitzer spectrum of the exoplanet HD189733b. Astrophys. J. 658, L115-L118 (2007)

15. Guyon, O., Pluzhnik, E.A., Galicher, R., Martinache, F., Ridgway, S.T., Woodruff, R.: APJ 622, 744 (2005)

16. Hebrard, G., Moos, H.W.: The Deuterium-to-oxygen ratio in the interstellar medium. Astrophys. J. 599, 297-311 (2003)

17. Hebrard, G., et al.: FUSE determination of a low deuterium abundance along an extended sight line in the galactic disk. Astrophys. J. 635, 1136-1150 (2005)

18. Herczeg, G.J., Wood, B.E., Linsky, J.L., Valenti, J.A., ohns-Krull, C.M.: APJ 607, 369 (2004)

19. Hyde, R.A.: Eyeglass: very large aperture diffractive telescopes. Appl. Opt. 38(19), 4198-4212, 1 July (1999)

20. Karovska, M., Hack, W., Raymond, J., Guinan, E.: APJ 482, L175 (1997)

21. Karovska, M., Schlegel, E., Hack, W., Raymond, J.C., Wood, B.E.: APJ 623, L-137 (2005)

22. Karovska, M.: Astrophys. Space Sci. 304(1-4), 379-382 (2006)

23. Knutson, H.A., et al.: A map of the day-night contrast of the extrasolar planet HD189733b. Nature 447 183-186 (2007)

24. Koechlin, L., Perez, J.-P.: A limit in field-resolution ratio for interferometric arrays. In: Traub, W.A. (ed.) SPIE Proc vol. 4838, 411-415. Interferometry for optical astronomy, II Hawaii, aot (2002)

25. Koechlin, L., Serre, D., Duchon, P.: High resolution imaging with Fresnel interferometric arrays: suitability for exoplanet detection. Astron. Astrophys 443, 709-720 (2005) 
26. Lecavelier des Etangs, A., Vidal-Madjar, A., McConnell, J.C., Hebrard, G.: Atmospheric escape from hot Jupiters. Astron. Astrophys. 418, L1-L4 (2004)

27. Lehner, N., et al.: Far ultraviolet spectroscopic explorer survey of the local interstellar medium within 200 parsecs. Astrophys. J. 595, 858-879 (2003)

28. Massonnet, D.: Brevet C. N. E. S.: un nouveau type de tèlescope spatial, Ref. 03.13403 (2003)

29. Moos, H.W., et al.: Overview of the far ultraviolet spectroscopic explorer mission. Astrophys. J. Lett. 538, L1-L6 (2000)

30. Morel, S., Koechlin L.: Exp. Astron. 7, 117-127 (1997)

31. Richardson, L.J., et al.: A Spitzer infrared radius for the transiting extrasolar planet HD209458b. Astrophys. J. 649, 1043-1047 (2006)

32. Richardson, D.L., et al.: A spectrum of an extrasolar planet. Nature 445, 892-895 (2007)

33. Serre, D., Koechlin, L., Deba, P.: Fresnel interferometric arrays for space-based imaging: testbed results. In: Proceeding of the SPIE, 6687-OI (2007)

34. Schupmann, L.: Die medial Fernrohre: Eine Neue Konstruktion for Grosse Astronomisch Instrumente. B.G Teubner, Leipzig (1899)

35. Sembach, K.R., et al.: The deuterium-to-hydrogen ratio in a low-metallicity cloud falling onto the milky way, Astrophys. J. Suppl. Ser. 150, 387-415 (2004)

36. van Dishoeck, E.F., Jonkheid, B., van Hemert, M.C.: Faraday Discuss. 133, 231 (2006)

37. Vidal-Madjar, A., et al.: An extended upper atmosphere around the extrasolar planet HD209458b. Nature 422, 143-146 (2003)

38. Soret, J.L.: Ann. Phys. Chem. 159, 320-337 (1875)

39. Vidal-Madjar, A., et al.: Detection of oxygen and carbon in the hydrodynamically escaping atmosphere of the extrasolar planet HD209458b. Astrophys. J. Lett. 604, L69-L72 (2004) 